\title{
O impacto da sobrecarga de trabalho e a satisfação do trabalhador em saúde mental
}

\section{The impact of overloading work and the satisfaction of workers in metal health}

\author{
Guilherme Correa Barbosa, Thayná Santos Buesso
}

Como citar este artigo: Barbosa, CC; Buesso, TS. $\bigcirc$ impacto da sobrecarga de trabalho e a satisfação do trabalhador em saúde mental. Revista Saúde (Sta. Maria). 2019 45 (2).

\section{Autor correspondente:}

Nome: Guilherme Correa Barbosa

E-mail: g.barbosa@unesp.br

Telefone: +55/|438801738

Formação Profissional: Enfermeiro

- Professor Assistente Doutor do

Departamento de Enfermagem

- Faculdade de Medicina de

Botucatu - FMB - UNESP

Filiação Institucional: Departamento de Enfermagem - Faculdade de Medicina de Botucatu - FMB - UNESP

Endereço para correspondência: Rua: Av. Prof. Mário Rubens Guimarães Montenegro $n^{\circ}: s / n$ Bairro: Botucatu Cidade: São Paulo Estado: São Paulo CEP: 18618687

Data de Submissão: 24/10/2017

Data de aceite:

1 1/07/2019

Conflito de Interesse: Não há conflito de interesse

\section{(cc) $\mathrm{B} Y-\mathrm{NC}-\mathrm{ND}$}

\section{RESUMO}

A pesquisa buscou descrever o impacto da sobrecarga de trabalho sobre a satisfação profissional em trabalhadores do Centro de Atenção Psicossocial (CAPS) de um município no interior do Estado de São Paulo. Estudo do tipo descritivo, exploratório, transversal com trabalhadores de um serviço de saúde mental comunitário. Foram aplicados um questionário sociodemográfico, a Escala de Avaliação da Satisfação de Profissionais em Serviços de Saúde Mental (SATIS-BR) e a Escala de Avaliação da Sobrecarga de Profissionais em Serviços de Saúde Mental (IMPACTO-BR). A análise foi através de procedimentos estatísticos. Os resultados deste estudo demonstraram que a avaliação dos trabalhadores sobre o CAPS se situa na faixa intermediária com relação aos aspectos avaliados no serviço, e apresenta uma avaliação com valores baixos de sobrecarga em relação ao trabalho no serviço. Os resultados do estudo estão de acordo com outras pesquisas publicadas, aponta bons resultados e afirma a importância destes serviços na comunidade fortalecendo a assistência aos pacientes e a qualidade de vida dos profissionais.

PALAVRAS-CHAVE: Pesquisa sobre Serviços de saúde; Saúde mental; Serviços Comunitários de Saúde Mental.

\section{ABSTRACT}

The research sought to describe the impact of work overload on job satisfaction in Psychosocial Care Center workers (CAPS) of a municipality in the state of São Paulo. A descriptive, exploratory, crosssectional study with workers from a Mental Health care service. A sociodemographic questionnaire was applied, the Evaluation Scale for the Satisfaction of Professionals in Mental Health Services (SATIS$B R$ ) and the Evaluation Scale for the Overload of Professionals in Mental Health Services (IMPACTO$\mathrm{BR})$. The analysis was through statistical procedures.The results showed that the evaluation of employees on CAPS is located in the middle range with respect to the aspects evaluated in the service, and presents an evaluation with low overhead values in relation to work in the service. Conclusions: The results of the study are in accordance with other published research, points out good results and affirms the importance of these services in the community strengthening the assistance to the patients and the quality of life of the professionals.

KEYWORDS: Mental Health; Health Services Research; Community Mental Health Research. 


\section{INTRODUÇÃO}

A atenção à saúde mental tem buscado a implantação de serviços substitutivos ao hospital psiquiátrico, com destaque aos Centros de Atenção Psicossocial (CAPS). Essa modalidade de atendimento foi implantada em diversos municípios brasileiros em substituição aos hospitais psiquiátricos, atendendo ao que é preconizado pela Reforma Psiquiátrica Brasileira. Estes serviços constituem-se em um sistema comunitário de saúde mental e tem como uma de suas metas a inclusão social da pessoa com transtorno mental' ${ }^{1}$.

Neste contexto, os CAPS assumem relevância no cenário das novas práticas em saúde mental no país. Essa mudança paradigmática deve englobar a relação que se estabelece com o usuário, equipe e família, e entre esses e a comunidade. A mudança de papéis, a democratização das instituições, o envolvimento e responsabilização da comunidade devem somar-se ao tratamento. O cuidado na comunidade torna-se mais complexo, interdisciplinar exigindo que as práticas e saberes tradicionais sejam reconstruídos para responder à esta transformação.

Com a Portaria 336/2002, se inicia a implantação dos Centros de Atenção Psicossocial, que passa a ser considerado um dos pilares para a construção de uma rede de serviços direcionada as pessoas com transtornos mentais. De acordo com as propostas do Ministério da Saúde, estes dispositivos devem utilizar o cuidado na comunidade, buscando atender às necessidades de seus usuários de forma sistemática e ativa, coadunando com o meio cultural e a comunidade em que estão inseridos, e de acordo com os princípios da Reforma Psiquiátrica².

Com mais de uma década da implantação destes serviços, torna-se uma necessidade realizar processos avaliativos dos mesmos. Estes processos devem monitorar as suas ações e acompanhar suas práticas de forma a construir um serviço que se proponha a atender a inserção do usuário, com maior resolubilidade, com uma intervenção pautada na diversidade de saberes de uma equipe multiprofissional e na utilização de recursos múltiplos.

Porém, os serviços substitutivos têm apresentado, em diversos países, entraves que dificultam o atendimento adequado aos pacientes e colocam em risco a meta da reinserção social e qualidade de vida, tais como: número insuficiente de serviços, falta de recursos humanos e materiais, despreparo dos profissionais, falta de integração entre serviços, conflitos entre profissionais e falta de repasse de verba ${ }^{3}$. Por causa desses impasses, a prática da avaliação contínua desses serviços se torna uma necessidade para que possa atingir a qualidade almejada.

O nível de satisfação dos trabalhadores é um dos indicadores de qualidade dos serviços de saúde mental ${ }^{4}$. Apresenta uma avaliação positiva do profissional sobre diversos aspectos do serviço, como relacionamento com colegas, qualidade do tratamento oferecido aos usuários, condições de trabalho, aspectos estruturais e organizacionais, e participação e envolvimento nas decisões tomadas no serviço. Um baixo nível de satisfação dos profissionais no trabalho pode afetar o relacionamento com os pacientes e interferir no próprio tratamento oferecido 5 .

Outro indicador de qualidade do serviço é a avaliação dos níveis de sobrecarga dos trabalhadores. A nova 
forma de atendimento das pessoas com transtornos mentais, que ampliou a gama de cuidados necessários à integração destes, requer maior envolvimento dos profissionais com o serviço, o que pode resultar em maior sobrecarga ${ }^{6}$. Este envolve aspectos psicológicos, emocionais e físicos causados por sentimentos de pressão relacionados com demanda excessiva no trabalho, frustração com o trabalho, cansaço, medo de agressão e desejo de mudanças de emprego 5 . Trata-se de um elemento importante a ser aferido no processo avaliativo de um serviço de saúde mental, uma vez que se relaciona inversamente com a satisfação no serviço ${ }^{7,10}$. No trabalho de profissionais da saúde, sobrecarga e estresse acarretam repercussões pessoais, clínicas e organizacionais ${ }^{4}$.

A sobrecarga e insatisfação podem afetar a saúde geral do trabalhador, incluindo sua saúde mental, e acarretar danos não apenas em sua vida profissional, mas também nos aspectos sociais e comportamentais ${ }^{7,11}$. Também pode acarretar em maior frequência de absenteísmo, rotatividade e estresse, que podem comprometer a qualidade do atendimento prestado ${ }^{6}$.

A sobrecarga pode resultar do efeito de diversas variáveis, tais como manejo diário e prolongado com pessoas com transtornos mentais, responsabilidade para com a vida do paciente, dificuldade no estabelecimento de limites nas interações profissionais, manutenção da relação terapêutica, envolvimento excessivo com o trabalho e falta de gratificação financeira e social ${ }^{12}$.

Para isso, a hipótese deste estudo considera que o impacto da sobrecarga de trabalho predispõe menor qualidade de vida, que acarreta menor satisfação profissional, mesmo quando controlado para a presença de sintomatologia psiquiátrica. Portanto, esta pesquisa buscou descrever o impacto da sobrecarga de trabalho sobre a satisfação profissional dos trabalhadores de saúde mental do Centro de Atenção Psicossocial de um município no interior do Estado de São Paulo.

Este artigo apresenta relevância cientifica e social pois auxiliará a mapear as potencialidades e fragilidades dos serviços comunitários em saúde mental possibilitando melhorar a assistência aos usuários do serviço.

\section{MÉTODO}

Trata-se de um estudo do tipo descritivo, exploratório e transversal. Foi realizado em um Centro de Atenção Psicossocial (CAPS) de um município no interior do Estado de São Paulo em funcionamento desde 2002. Os dados foram coletados durante o período de maio e julho de 2015. O CAPS possui uma equipe formada de 38 profissionais, sendo: quatro psicólogos, quatro médicos, três enfermeiras, 12 técnicos e auxiliares de enfermagem, sete auxiliares de saúde, dois terapeutas ocupacionais, um auxiliar administrativo, um auxiliar de limpeza, dois farmacêuticos e duas assistentes sociais, sendo que uma atua como coordenadora do serviço. Definiu-se como critérios de inclusão: ser 
trabalhador no CAPS com no mínimo um ano de trabalho no serviço.

Para coleta de dados foram utilizados três instrumentos: um questionário sociodemográfico, a Escala de Avaliação da Satisfação de Profissionais em Serviços de Saúde Mental (SATIS-BR) e a Escala de Avaliação da Sobrecarga de Profissionais em Serviços de Saúde Mental (IMPACTO-BR). As escalas SATIS-BR e IMPACTO-BR integram um conjunto de escalas para avaliação de serviços de saúde mental, elaboradas pela Divisão de Saúde Mental da OMS, que foram validadas para o Brasil por Bandeira et al ${ }^{8,13}$. O questionário sociodemográfico continha as seguintes variáveis: sexo, idade, etnia, estado civil, qual seu maior grau de escolaridade, pós-graduação, há quanto tempo trabalha em CAPS, há quanto tempo trabalha neste CAPS, profissão, salário, carga horária, tipo de vínculo empregatício, possui outro vínculo empregatício.

A SATIS-BR avalia o grau de satisfação da equipe de profissionais em serviços de saúde mental. Possuiu 32 itens, sendo agrupados em quatro fatores que avaliam o grau de satisfação da equipe em relação à: 1. Qualidade dos serviços oferecidos aos pacientes, 2. Participação da equipe no serviço, 3. Condições de trabalho e 4. Relacionamento no serviço, incluídos na escala global. As alternativas de resposta aos itens estão dispostas em uma escala do tipo Likert com 5 pontos, na qual 1 representa que o profissional está muito insatisfeito e 5 que ele está muito satisfeito. Há também três questões qualitativas no final da escala, que avalia se os profissionais possuem alguma observação a fazer, que não tenha sido coberta pelas questões da escala. As três questões avaliam: o que o profissional achou melhor no serviço, o que ele achou pior e o que ele acha que deveria ser melhorado.

A IMPACTO-BR avalia a sobrecarga sentida pelos profissionais de saúde mental, em consequência do trabalho diário com pessoas portadoras de transtornos mentais. 0 questionário completo inclui 34 questões, dentre as quais apenas 18 constituem as questões quantitativas da escala propriamente dita, avaliando as repercussões do trabalho em diversos aspectos da vida do profissional. As alternativas de resposta estão dispostas em escala tipo Likert de 5 pontos, em que 1 = de forma alguma e 5 = extremamente. Estão agrupados em três fatores que avaliam: 1. Efeitos sobre a saúde física e mental, 2. Efeitos no funcionamento da equipe e 3. Sentimento de estar sobrecarregado, incluídos na escala global.

O projeto foi submetido ao Comitê de Ética em Pesquisa da Faculdade de Medicina de Botucatu da Universidade Estadual "Júlio de Mesquita Filho", seguindo as determinações da Resolução 466/12, do Conselho Nacional de Ética em Pesquisa - CONEP/CNS e aprovado com o n 972.320/2015.

Foi pedida autorização para o Comitê de Pesquisa do CAIS "Professor Cantídio" e para coordenação do CAPS. Alguns procedimentos éticos que antecedem à coleta de dados foram cumpridos, como se segue: Contato por meio telefônico com a coordenação do CAPS, para o agendamento de uma reunião com a finalidade de apresentar o projeto 
e a proposta da pesquisa. Após esse primeiro contato, as visitas foram agendadas em dias e horários que eram mais convenientes ao funcionamento do serviço. Em seguida, os trabalhadores foram informados acerca da realização da pesquisa, e para participação da pesquisa, e todos igualmente assinaram os TCLE. Com a obtenção dos TCLE dos participantes, deu-se início à coleta de dados.

O banco de dados foi construído através da codificação dos dados pelo pesquisador, em seguida, processados no software Microsoft Excel® 2010, por meio de dupla digitação, com a finalidade de minimizar os possíveis erros. Os dados foram processados por meio do programa SPSS, versão 13.0, envolvendo a análise descritiva dos dados, testes inferenciais de médias e construção de intervalos de confiança para cada uma das variáveis quantitativas.

\section{RESULTADO}

Descrição da amostra

A população total de funcionários no serviço foi de 38 pessoas, sendo que três não preenchiam os requisitos da pesquisa, outros quatro estavam de licença e dois não quiseram participar. Com isso, tivemos uma participação de 29 entrevistados.

O perfil dos participantes está descrito na distribuição de frequências dos dados (Tabela 1) segundo idade, tempo de trabalho em CAPS, tempo de trabalho neste CAPS, carga horária, sexo, etnia, estado civil, escolaridade, profissão, salário, vínculo, outro vínculo.

Tabela 1. Apresentação das informações sociodemográficas segundo os profissionais de saúde do CAPS ( $n=29$ ), 2015.

\begin{tabular}{|c|c|c|}
\hline Variáveis quantitativas & Média & Mediana \\
\hline Idade & 42,3 & 40 \\
\hline Tempo em CAPS & 3,6 & 2 \\
\hline Tempo neste CAPS & 2,9 & 2 \\
\hline Carga Horária & 28,9 & 30 \\
\hline Variáveis categóricas & $\mathbf{N}$ & $\%$ \\
\hline \multicolumn{3}{|l|}{ Sexo } \\
\hline Masculino & 4 & 13,8 \\
\hline Feminino & 25 & 86,2 \\
\hline \multicolumn{3}{|l|}{ Etnia } \\
\hline Branca & 23 & 79,3 \\
\hline Negra & 1 & 3,4 \\
\hline
\end{tabular}




\begin{tabular}{lcc} 
Pardo & 5 & 17,2 \\
\hline Profissão & 3 & \\
\hline Médico & 3 & 10,3 \\
\hline Enfermeiro & 3 & 10,3 \\
\hline Psicólogo & 2 & 10,3 \\
\hline Assistente social & 9 & 31,0 \\
\hline Técnico/auxiliar de enfermagem & 2 & 6,9 \\
\hline Terapeuta ocupacional & 1 & 3,4 \\
\hline Farmacêutico & 6 & 20,7 \\
\hline Outro & & \\
\hline Vínculo & 1 & 3,4 \\
\hline CLT & 22 & 75,8 \\
\hline Estatutário & 6 & 20,7 \\
\hline Outros & & \\
\hline Outro vínculo & 19 & 65,5 \\
\hline Não & 10 & 34,5 \\
\hline Sim & & \\
\hline
\end{tabular}

Fonte: Dados da pesquisa, 2015.

Na distribuição dos trabalhadores, há um predomínio do perfil feminino, $86,2 \%$, que se configura normalmente em todas as outras áreas da saúde. Apresentou média de idade de 42,3 anos, mediada de 40 anos sendo o mínimo de 26 e a máxima de 59 , configurando uma população de meia idade. Em relação à cor da pele, $79,3 \%$ dos entrevistados se dizem brancos, $62,1 \%$ referiram possuir uma relação estável ou casamento e $21,4 \%$ são solteiros.

Dos entrevistados, $31 \%$ eram de técnico/auxiliares de enfermagem, sendo a categoria profissional em maior número no serviço, e a predominância de tempo no CAPS era de 2 a 4 anos, perfazendo 41,38\%. Quanto à formação e escolaridade, a maioria dos trabalhadores possui ensino superior, $72,4 \%$, sendo que cerca de $51,7 \%$ possuem pósgraduação.

No item salarial, o que destaca é que $72,4 \%$ ganha até três salários mínimos, tendo $89,6 \%$ dos profissionais uma jornada de trabalho de 30 horas semanais. No vínculo empregatício temos $95,7 \%$ dos profissionais em regime estatutário. Apenas $34,5 \%$ dos profissionais apresentam mais de um vínculo empregatício.

Satisfação dos trabalhadores com o serviço de Saúde Mental

Para descrição de satisfação dos trabalhadores utilizou-se a escala SATIS-BR, apresentada na tabela a seguir, seguindo as questões validadas no Brasil por Bandeira, Pitta e Mercier ${ }^{8}$. A aplicação da escala aos trabalhadores permitiu a identificação do quadro global de satisfação, conforme os fatores e escala global apresentados abaixo, que indica uma 
avaliação intermediária de todos os itens com média em torno de 3,4 (dp 0,6) em uma escala tipo likert de 1 a 5.

A tabela apresenta os resultados descritivos dos escores de satisfação global e por fatores $(1,2,3,4)$ dos trabalhadores para cada item da escala SATIS-BR, em termos das médias e desvios-padrão ( $n=29)$.

Tabela 2. Medidas descritivas dos escores da escala SATIS-BR, 2015.

\begin{tabular}{lcccc} 
Item da Escala & Média & $\begin{array}{c}\text { Desvio } \\
\text { padrão }\end{array}$ & $\begin{array}{c}\text { Escore } \\
\text { Mínimo }\end{array}$ & $\begin{array}{c}\text { Escore } \\
\text { Máximo }\end{array}$ \\
\hline Global do SATIS BR & 3,4 & 0,6 & 1,7 & 4,4 \\
\hline Qualidade da assistência prestada ao usuário & 3,7 & 0,7 & 2,1 & 4,9 \\
\hline Quanto à sua participação no serviço & 3,2 & 0,7 & 1,0 & 4,5 \\
\hline Condições de trabalho & 3,2 & 0,7 & 1,7 & 4,4 \\
\hline Relacionamento no serviço & 3,5 & 0,9 & 1,6 & 5,0 \\
\hline
\end{tabular}

Fonte: Dados da pesquisa, 2015.

O escore global do SATIS-BR teve média de 3,4 (dp 0,6), variando de 1,7 a 4,4, tendo um escore intermediário. As questões quanto à qualidade da assistência prestada ao usuário foram as que receberam melhor avaliação, com uma média de 3,7 (dp 0,7), com uma variação de 2,1 a 4,9. As questões referentes ao relacionamento no serviço receberam a avaliação com média de 3,5 (dp 0,9), variando entre 1,6 e 5,0.

As piores avaliações ficaram para quanto à sua participação no serviço e condições de trabalho. Temos uma média de score de 3,2 (dp 0,7) para quanto à sua participação no serviço, variando de 1,0 a 4,5. Já para as condições de trabalho temos uma média de score de 3,2 (dp 0,7), variando de 1,7 a 4,4.

Em relação à questão de existirem outros cuidados de que o usuário necessita, mas que não recebe no CAPS, 34,4\% referiu que com certeza há e especificaram: serviços de reabilitação (fonoaudiologia e fisioterapia), grupos e oficinas para resgate e/ou desenvolvimento de potencialidades, habilidades e competências; atendimento clínico, serviços de terapia, assistência social, habitação, acompanhantes terapêuticos e residências terapêuticas.

No quesito do momento em que os pacientes entram em contato com este serviço, $68,7 \%$ dos funcionários afirmaram que em geral existem outras opções disponíveis para eles, e especificaram: Unidade Básica de Saúde (UBS), CAPS I, Ambulatório de Saúde Mental, Estratégia de Saúde da Família (ESF) com apoio do NASF, Hospital das clínicas UNESP, Pronto Socorro, CAPS ad, Oficina Girassol e Hospital Dia.

Na questão de que se o profissional recomendaria o CAPS se tivesse um amigo/familiar que estivesse necessitando de ajuda de uma Unidade de Saúde Mental, 58,2\% afirmaram que com certeza indicariam, justificando: a facilidade de acesso, profissionais competentes, experientes e acolhedores; acolhimento diário, garantia de medicação, confiança na equipe e no serviço, atendimento multiprofissional, responsabilidade e ética com o sigilo, encaminhamentos e condutas adequadas. 
Em relação ao que os profissionais mais gostam no serviço, eles apontaram: o vínculo paciente/funcionário, a humanização do cuidado e os profissionais serem acolhedores, discussões de casos, acolhimento, eficácia dos tratamentos, aprendizado diário, atendimento psiquiátrico, visitas domiciliares, profissionalismo, grupos e oficinas.

Em relação ao que os profissionais menos gostam no serviço, indicam: a falta de autonomia falta de pessoal especializado e supervisão, falta de comprometimento de alguns profissionais que leva à sobrecarga em outros; falta de processo de trabalho, plano de carreira inexistente, falta de entrosamento na equipe, local de trabalho inadequado, falta de treinamentos e educação permanente; trabalho pouco valorizado, salários baixos, decisões hierárquicas, excesso de burocracia e falta de respaldo aos trabalhadores pela coordenação.

No item de que o serviço poderia ser melhorado, 89,6\% afirmaram que sim dando as seguintes sugestões: atividades para promover a interdisciplinaridade na equipe, supervisão, estrutura física adequada, introdução de educação permanente, seleção e treinamento de funcionários, contratação de mais profissionais, melhor remuneração, diminuir a burocracia e estreitar laços com outros serviços da Rede de Atenção Psicossocial (RAPS), planos de carreira, definir melhor o processo de trabalho, e espaços de lazer e convivência para os profissionais.

\section{Escala de Avaliação do Impacto do Trabalho em Serviços de Saúde Mental (IMPACTO-BR)}

Para descrição do impacto do trabalho em serviços de saúde mental utilizou-se a escala IMPACTO-BR e apresentada na tabela a seguir, seguindo as questões validadas no Brasil por Bandeira, Pitta e Mercier8. A aplicação da escala aos trabalhadores permitiu a identificação do quadro global do impacto, conforme os fatores e escala global apresentados abaixo que indica uma avaliação com valores baixos de sobrecarga de todos os itens com média em torno de 2,1 (dp 0,7), em uma escala tipo likert de 1 a 5.

A tabela a seguir apresenta os resultados descritivos dos escores do impacto global e por fatores $(1,2,3)$ dos trabalhadores para cada item da escala IMPACTO-BR, em termos das médias e desvios-padrão $(n=29)$.

Tabela 3. Medidas descritivas dos escores da escala IMPACTO-BR, 2015.

Item da Escala

\begin{tabular}{lllll}
\hline Global do SATIS BR & 2,1 & 0,7 & 1,0 & 3,7 \\
\hline Efeitos sobre a saúde física e mental (F1) & 2,0 & 0,9 & 1,0 & 4,0 \\
\hline Efeitos no funcionamento da equipe (F2) & 2,1 & 0,8 & 1,0 & 3,8 \\
\hline Sentimento de estar sobrecarregado (F3) & 2,2 & 0,8 & 1,0 & 4,0 \\
\hline
\end{tabular}

Média Desvio Escore Escore

padrão Mínimo Máximo

\footnotetext{
Fonte: Dados da pesquisa, 2015.
} 
A descrição realizada individualmente para cada um dos fatores buscou apresentar o grau de sobrecarga em diferentes dimensões do serviço. Conforme os valores das médias, o terceiro fator apresentou o maior grau de sobrecarga da equipe.

O escore global do IMPACTO-BR teve média de 2,1 (dp 0,7), variando de 1,0 a 3,7, indicando grau de sobrecarga entre "não muito" e "mais ou menos". Esse resultado indica que os profissionais apresentaram valores baixos de sobrecarga.

Segundo os valores das médias, o terceiro fator apresentou o maior grau de sobrecarga da equipe com média de 2,2 (dp 0,8), resultado relativo ao sentimento de estar sobrecarregado. As questões referentes aos efeitos no funcionamento da equipe receberam a avaliação com média de 2,1 (dp 0,8). 0 primeiro fator apontou o menor grau de sobrecarga equipe com média de 2,0 (dp 0,9), resultado referente aos efeitos sobre a saúde física e mental.

\section{DISCUSSÃO}

No serviço, a maioria dos profissionais é do sexo feminino, de cor branca, casado ou com companheiro, e a média de idade é de 42, 3\%, constituindo então uma população de meia idade. Esses resultados são compatíveis com outros estudos que também levantaram o perfil sociodemográfico dos profissionais da área da saúde, como Rebouças, Legay e Abelha, De Marco et al, Barbosa et al, Guimarães et al, bosque at al e Clementino et al $7,10,14,15,16,17$.

A maioria dos profissionais possuem ensino superior, $72,4 \%$ deles recebe até 3 salários mínimos, sendo que 95,7\% tem vínculo estatutário e 34,5\% tem segundo tipo de vínculo empregatício. É interessante observar que, a maioria dos profissionais do serviço é da equipe de enfermagem, e que também são eles que têm a maior presença de segundo vínculo empregatício.

Em um artigo de revisão da literatura, Melo, Barbosa e Souza analisaram 17 artigos publicados relacionados à questão da satisfação do profissional de enfermagem; e foi observado que a insatisfação salarial e desvalorização profissional são recorrentes ${ }^{18}$. Pode-se relacionar que, o que leva os profissionais a buscarem outro vínculo empregatício, seria para uma complementação da renda. Outro trabalho apontou que $77,6 \%$ apresenta vínculo empregatício por CLT ou caráter temporário Clementino et al ${ }^{17}$.

Os profissionais apresentaram na SATIS-BR escore médio global de 3,4, que pode ser considerado como intermediário; além disso, em nenhum dos outros fatores avaliados na escala ele obteve média acima de 3,7; o que significa que apesar dos níveis de satisfação estarem acima da média, existe a necessidade de introduzir melhorias no serviço.

Os dois fatores que obtiveram os maiores índices de satisfação foram a de qualidade prestada ao usuário, e a do relacionamento no serviço; e as de menor índice de satisfação foram às relacionadas com a participação do profissional 
no serviço e às condições de trabalho, resultados parecidos em pesquisas com mesmo públicos ${ }^{7,12,14,16,17}$. Analisando os dados das escalas juntamente com a parte descritiva, é evidente que o fator mais satisfatório para os funcionários é o vínculo com os usuários, em acordo com os resultados obtidos por Rebouças, Legay e Abelha7.

Apesar do fator de relacionamento no serviço ser uma das que obteve melhores índices de satisfação, é importante elencar que na parte descritiva, um dos maiores problemas levantados foi quanto ao entrosamento da equipe. Isso pode ser explicado levando em conta as próprias dificuldades por eles expostas, como a falta de pessoal especializado, falta de treinamento e de educação permanente; então por mais que os relacionamentos sejam bons, há um déficit no trabalho em equipe, consequentemente afetando a interdisciplinaridade.

Os resultados dessa escala, apesar de intermediários, têm potencial para serem melhorados; e 89,6\% dos funcionários acham que o serviço pode ser melhorado. Os baixos salários, déficit de recursos humanos, e de um local de trabalho adequado; dificuldades levantadas pela equipe, estão em concordância com os dados obtidos por Rebouças, Legay e Abelha, De Marco et al, Ishara e Clodoaldo et al; porém a falta de autonomia, de entrosamento na equipe e a falta de respaldo dos trabalhadores pela coordenação foram dificuldades levantadas nessa pesquisa, e que não tiveram semelhança comparando com as outras ${ }^{7,10,11}$.

Na escala IMPACTO-BR, o escore global foi de 2,1, que indica um baixo grau de impacto nos funcionários. Devese levar em conta que alguns profissionais não quiseram participar e outros estavam de licença, o que pode alterar o real impacto do serviço, em vista que os que mais sofrem podem ter ficado de fora da pesquisa.

O fator de maior escore foi de sentir se sobrecarregado (2,2), e o de menor escore foi o de efeitos sobre a saúde física e mental (2,0), resultados parecidos com os de Rebouças, Legay e Abelha; De Marco at al, e Leal, Bandeira e Azevedo $7,10,19,20$.

A literatura aponta que a sobrecarga e insatisfação podem afetar a saúde geral do trabalhador, incluindo sua saúde mental que acarreta danos em vários aspectos da vida do trabalhador envolvendo a profissional, social e comportamental. Tais profissionais lidam no cotidiano com o sofrimento psíquico, deixando o ambiente permeado por intensa produção subjetiva e intersubjetiva ${ }^{12,17}$.

Atentando-se ao fator que obteve o maior escore na IMPACTO-BR, o de sentir-se sobrecarregado; pode-se correlacionar com os problemas levantados na SATIS-BR, novamente citando as dificuldades de entrosamento da equipe. Podemos supor então que se houver melhor definição de papéis no serviço e se as atividades realizadas forem feitas de modo interdisciplinar, o impacto do serviço seria menor ainda.

\section{CONSIDERAÇÕES FINAIS}

Os resultados da pesquisa demonstraram que os níveis de satisfação e impacto nos profissionais do serviço 
estão em concordância com outros artigos já publicados, porém não deve ser generalizado para outros serviços visto que as condições de trabalho e relações no serviço são diferentes.

É importante também que sempre haja a avaliação do serviço por parte dos profissionais, para que se consiga desenvolver métodos de levantamento de demandas e melhor resolução das mesmas; desconstruir o ambiente de trabalho e as relações para conseguir um bom trabalho multiprofissional e consistência na interdisciplinaridade.

Como este trabalho foi realizado em um único serviço de saúde mental, não pode ser generalizado para outros serviços. Deste modo, espera-se que os resultados apresentados orientem a política local na elaboração de novas ações que ampliem a atenção aos usuários, bem como a sua permanência no tratamento, no sentido de promover a sua reinserção social e a diminuição dos sintomas apresentados.

\section{REFERÊNCIAS}

1. Amarante P. Saúde Mental, Desinstitucionalização e Novas Estratégias de Cuidado. In: Políticas e Sistema de Saúde no Brasil cap.20 (org) Ligia Giovanella et al. Editora Fiocruz/ CEBES , 2008.

2. Brasil, Ministério da Saúde. Saúde Mental no SUS: Os Centros de Atenção Psicossocial. Brasília, 2004.

3. Andreoli SB. Serviços de saúde mental no Brasil. In: Mello MF, Mello AAF, Kohn R. (Org.). Epidemiologia da saúde mental no Brasil. Porto Alegre: Artmed, 2007.

4. Borges LO. et al. A síndrome de Burnout e os valores organizacionais: um estudo comparativo em hospitais universitários. Psicol Reflex Crít, 2002; 45(1): 189-200.

5. Ishara S, Bandeira M, Zuardi AW. Public psychiatric services: job satisfaction evaluation. Rev Bras Psiquiatr, 2008; 30(1): 38-41.

6. Rebouças D. et al. O trabalho em saúde mental: um estudo de satisfação e impacto. Cad Saúde Pública, 2008; 24(3): 624-32.

7. Rebouças D, Legay LF, Abelha L. Satisfação com o trabalho e impacto causado nos profissionais de serviço 
de saúde mental. Revista Saúde Pública, 2007; 41(2): 244-50.

8. Bandeira M, Pitta AMF, Mercier C. Escalas brasileiras de avaliação da satisfação (SATIS-BR) e da sobrecarga (IMPACTO-BR) da equipe técnica em serviços de saúde mental. J. Bras. Psiquiatr, 2000; 49(4): 105-15.

9. Bandeira M, Ishara S, Zuardi AW. Satisfação e sobrecarga de profissionais de saúde mental: validade de construto das escalas SATIS-BR e IMPACTO-BR. J Bras Psiquiatr, 2007; 56(4): 280-86.

10. De Marco PF et al. O impacto do trabalho em saúde mental: transtornos psiquiátricos menores, qualidade de vida e satisfação profissional. J Bras Psiquiatr, 2008; 57(3): 178-83.

11. Ishara S. Equipes de saúde mental: avaliação da satisfação e do impacto do trabalho em hospitalização parcial. 2007. Tese (Doutorado em Saúde Mental)-Universidade de São Paulo, Ribeirão Preto, 2007.

12. Pelisoli C, Moreira ÂK, Kristensen $\mathrm{CH}$. Avaliação da satisfação e do impacto da sobrecarga de trabalho em profissionais de saúde mental. Mental, 2007; 5(9): 63-79.

13. Bandeira M, Pitta AMF, Mercier C. Escalas da OMS de avaliação da satisfação e da sobrecarga em serviços de saúde mental. J Bras Psiq, 1999; 48(6): 233-44.

14. Barbosa GB et al. Trabalho e saúde mental dos profissionais da Estratégia Saúde da Família em um município do Estado da Bahia, Brasil. Rev Bras Saúde Ocup, 2012; 17(126): 306-15.

15. Guimarães JMX, Jorge MSB, Assis MMA. (In)satisfação com o trabalho em saúde mental: um estudo em Centros de Atenção Psicossocial. Ciência \& Saúde Coletiva, 2011; 16(4): 2145-54.

16. Bosque RM, Oliveira MAF de, Silva NN da, Claro HG, Fernandes IFAL. Satisfação dos trabalhadores, usuários e familiares dos Centros de Atenção Psicossocial em álcool e outras drogas. Rev enferm UFPE on line., Recife, 2017; 11(Supl. 11):4598-606.

17. Clementino F, Miranda F, Martiniano C, Marcolino E, Pessoa-Júnior J, Fernandes N. Avaliação da satisfação 
e sobrecarga de trabalho dos trabalhadores dos Centros de Atenção Psicossocial. Revista de Pesquisa: Cuidado é Fundamental Online, 2018;10(1): 153-159.

18. Melo MB, Barbosa MA, Souza PR. Satisfação no trabalho da equipe de enfermagem: revisão integrativa. Rev Latino-Am Enfermagem, 2011; 19(4): 1047-55.

19. Clodoaldo SS, Barbosa GC, Oliveira MAF de. Satisfação dos trabalhadores de um centro de atenção psicossocial em álcool e outras drogas. Revista UNINGÁ, 2017; 52(1): 12-17.

20. Leal RMAC, Bandeira MR, Azevedo KRN. Avaliação da qualidade de um serviço de saúde mental na perspective do trabalhador: satisfação, sobrecarga e condições. Psicol. teor. prat., 2012; 14(1): 15-25. 